\title{
El deterioro de las piedras graníticas de la fachada de la catedral de Vila Real ( $\mathrm{N}$ de Portugal)
}

\author{
Deterioration of the granitic stones of main front \\ of the Vila Real Cathedral ( $N$ of Portugal)
}

\author{
Machado, B. ${ }^{(*)}$, Gomes, M. E. P. ${ }^{*}$, Suárez del Río, L. M.(**)
}

Recepción/Received: 10-II-2007

Aceptación/Accepted: 12-XI-2008

Publicado online/Online publishing: 20-XI-2009

\section{RESUMEN}

La Catedral de Vila Real (Norte de Portugal) se encuentra en el centro de la ciudad y se han utilizado, al menos, cuatro granitos de dos micas para su construcción extraídos de sus alrededores. El estudio petrográfico de estos granitos pone de manifiesto que poseen un diferente grado de alteración. La determinación de algunas propiedades físicas de los granitos -porosidad abierta, densidad aparente y real, absorción libre de agua, porometría y capilaridad- confirman los distintos grados de alteración. La catedral ha sufrido una reciente limpieza y sobre las piedras de la Catedral se observan distintas formas de alteración, sobre todo desagregación granular (arenización), escasas pátinas finas negras, desplacaciones, escamas y costras negras, especialmente en la fachada principal, expuesta al SW. El fenómeno de la desagregación granular es el más importante y es el responsable de de la pérdida de material en las piedras más afectadas. Su génesis se relaciona con la presencia de pequeñas cantidades de sales solubles, como halita y nitratos, y a la exposición al sol.

Palabras clave: catedral Vila Real, granito, alteración, deterioro, sales solubles, halita.

\section{SUMMARY}

The cathedral of Vila Real (North of Portugal) is located in the city centre and at least four regional two-mica granites were used in its construction. A petrographic study of these granites concluded that the stones had different degrees of alterations. The identification of some physical properties of the granites -open porosity, real and apparent density, free water absorption, porometry and capillary transmissionconfirms their susceptibility to degrees of weathering. A recent cleaning intervention was made and several types of deterioration were visible in the monument,s stones, namely: granular disintegration, some thin black layers, plates, flakes and black crusts, especially in the main front exposed to SW. Granular disintegration is the most important deterioration process and is responsible for the loss of material in the most-affected stones; it is also related to the presence of small amounts of highly soluble salts such as halite and nitrates and to solar exposure.

Keywords: Vila Real cathedral, granite, alteration, deterioration, soluble salts, halite

\footnotetext{
(*) Universidade de Trás-os-Montes e Alto Douro, Vila Real (Portugal)

(**) Universidad de Oviedo (Oviedo, España).
} 


\section{INTRODUCCIÓN}

La ciudad de Vila Real se encuentra en la Zona Centro Ibérica, que constituye una de las principales unidades geotectónicas del Macizo Ibérico, y que se extiende por la zona occidental y central de la península Ibérica. Desde el punto de vista litoestratigráfico, en los alrededores de la ciudad de Vila Real afloran rocas metasedimentarias pertenecientes al Grupo del Duero, representado por las Formaciones de Desejosa y de Pinhao, de edad Cámbrico Medio. El centro histórico de la ciudad se asienta sobre granitos hercínicos (320-300 M.a.) (1).

La seo catedral de Vila Real se sitúa en el centro histórico de la ciudad, en el norte de Portugal (Figura 1). El monumento fue construido en el siglo $\mathrm{XV}$, pero algunas partes han sido añadidas en el siglo XVIII. En su construcción se han utilizado al menos cuatro tipos de granitos que afloran en los alrededores de la ciudad (1) y que se encuadran en las siguientes tipologías: granitoides sintectónicos (granitos de Vale das Gatas-VG y granito de Paredes-Pa) y granitoides tardi a post-orogénicos (granito de Lamares-La y granito de Benagouro-Be). La distribución de estos materiales en la fachada de la catedral se representa en la Figura 2.

Desde el punto de vista climático, a partir de los datos registrados entre los años 1958 y 1988, puede decirse que el período seco es corto, ciñéndose apenas a los meses de julio y agosto, siendo el mes de julio el más

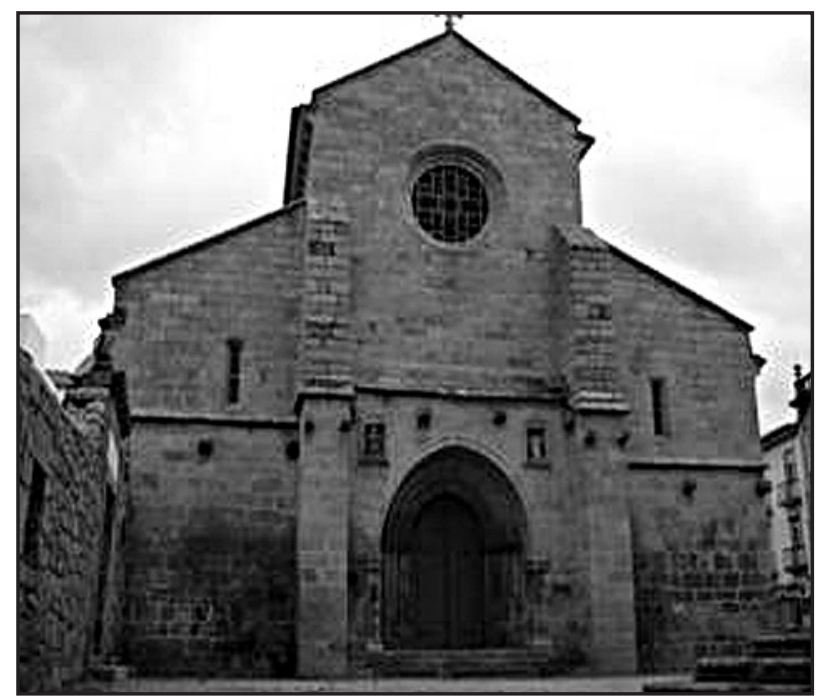

Figura 1. Fachada principal (W) de la seo catedral de Vila Real. Figure 1. Main entrance (W) of Vila Real Cathedral.

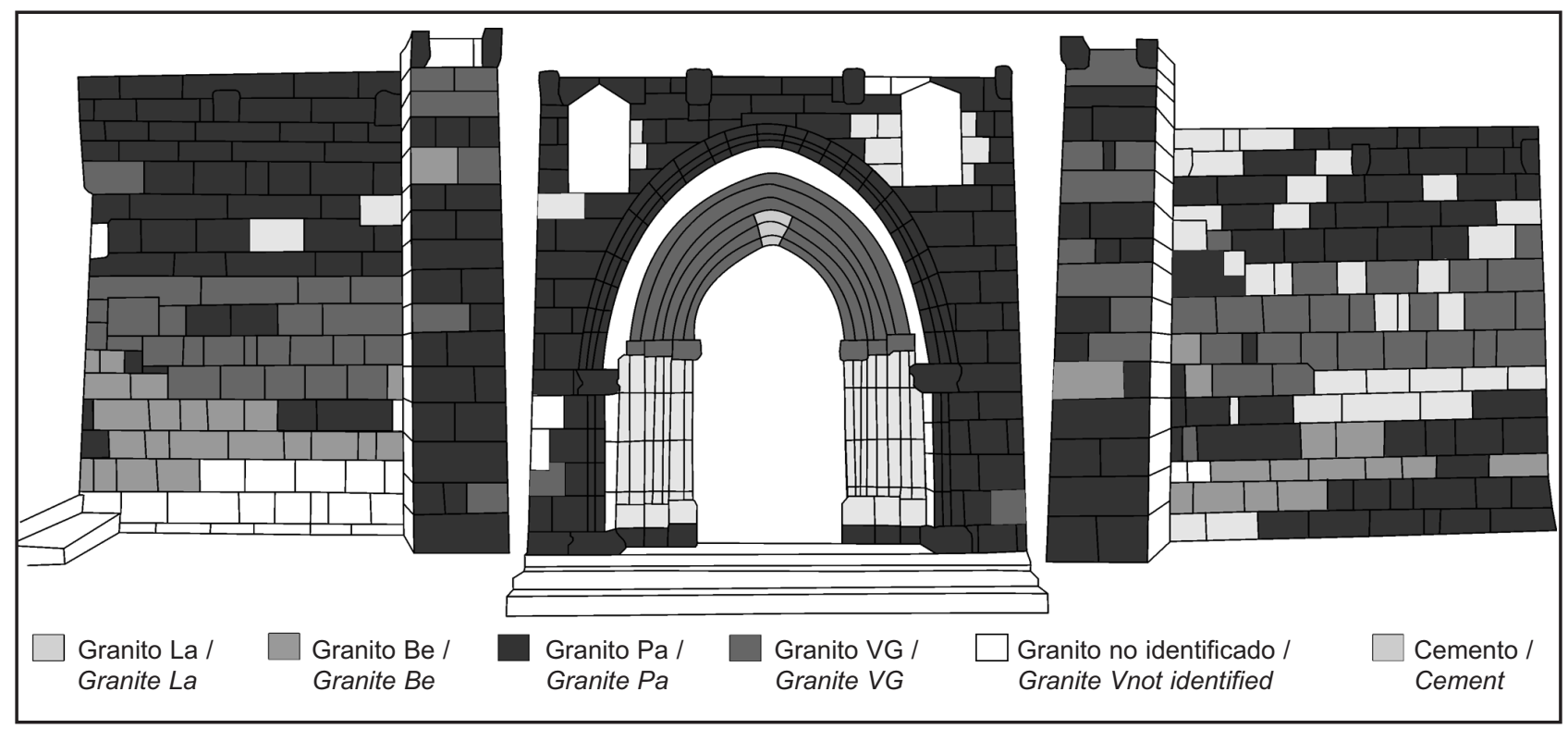

Figura 2. Cartografía de los cuatro tipos de granitos presentes en la fachada principal (W) de la seo catedral. Figure 2. Mapping of lithologies present in the main entrance $(W)$ of cathedral. 
caliente del año, con temperaturas medias de $21,4^{\circ} \mathrm{C}$, y el mes de enero el más frío, con temperaturas medias de $6,4^{\circ} \mathrm{C}$. Este mes también se caracteriza por tener como media el mayor número de días -9- con heladas. La temperatura máxima registrada, en un mes de julio, fue de $35,2{ }^{\circ} \mathrm{C}$ y la mínima, en enero, de $-2,6^{\circ} \mathrm{C}$. Los meses de enero y diciembre son los más húmedos y menos soleados. Los valores medios de humedad relativa oscilan entre el 74 y $90 \%$. Respecto a las precipitaciones, las más abundantes son en diciembre, alcanzando valores medios de $165 \mathrm{~mm}$, y las menos intensas, en el mes de julio, registrándose valores medios de $23,8 \mathrm{~mm}$.

Vila Real no es una ciudad polucionada y los datos atmosféricos muestran que el contenido de dióxidos de azufre y nitrógeno y de partículas en el aire no es importante. Los valores máximos de partículas $\mathrm{PM}_{10}$ en suspensión, dióxido de nitrógeno y dióxido de azufre medidos durante una semana ( 16 al 22 de septiembre de 2003) en la zona de la catedral de Vila Real, respectivamente, fueron de $52 \mu \mathrm{g} / \mathrm{m}^{3}, 108 \mu \mathrm{g} / \mathrm{m}^{3}$ y $31 \mu \mathrm{g} / \mathrm{m}^{3}$ (2).

El monumento sufrió un proceso de conservación y limpieza en los años 2001 a 2004, para la eliminación de un olivo que crecía en la torre y de algunos revocos, utilizando solamente agua a presión.

\section{PARTE EXPERIMENTAL}

En el presente estudio se ha realizado una cartografía de los distintos tipos de granitos presentes en la catedral de Vila Real y se han caracterizado desde el punto de vista petrográfico y petrofísico, con el fin de intentar explicar los fenómenos de alteración que se observan en el monumento. Para ello, se han cartografiado las formas de alteración de la fachada y se han relacionado con los tipos de granitos con los que ha sido construida. Además, se han estudiado las sales solubles extraídas de algunas zonas de la portada, para identificar su naturaleza y peligrosidad en los procesos de deterioro.

El estudio petrográfico de los granitos se ha realizado sobre láminas delgadas pulidas en el microscopio petrográfico y en el microscopio electrónico de barrido (MEB). El microscopio de barrido es un SEM/ESEM FEI Quanta 400 con EDS de EDAX. Algunas muestras del material desagregado, recogido después de la etapa de limpieza en una columna en avanzado estado de degradación debido a la arenización, también fueron estudiadas a través de un MEB equipado con un sistema de microanálisis. Las imágenes se obtuvieron mediante la detección simultánea de electrones secundarios y retrodispersados. Para analizar algunos minerales se usó la microsonda electrónica Cameca Camebax del Laboratório Nacional de Energia e Geologia (LNEG) de São Mamede de
Infesta. El potencial de aceleración fue de $15 \mathrm{kV}$ y la intensidad de emisión de 20 nA. La metalización de las láminas pulidas se hizo con grafito y se utilizaron los siguientes patrones: ortoclasa ( $\mathrm{Si}-\mathrm{K} \alpha$, Al-K $\alpha$ e $\mathrm{K}-\mathrm{K} \alpha)$, $\mathrm{MnTiO}_{3}(\mathrm{Ti}-\mathrm{K} \alpha$ e $\mathrm{Mn}-\mathrm{K} \alpha), \mathrm{Fe}_{2} \mathrm{O}_{3}(\mathrm{Fe}-\mathrm{K} \alpha), \mathrm{MgO}(\mathrm{Mg}-\mathrm{K} \alpha)$,

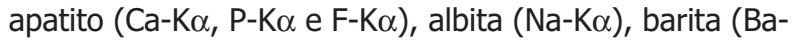
$\mathrm{K} \alpha), \mathrm{Cr}_{2} \mathrm{O}_{3}(\mathrm{Cr}-\mathrm{K} \alpha)$ y vanadinita $(\mathrm{Cl}-\mathrm{K} \alpha)$.

Para la realización de los ensayos de propiedades físicas se utilizaron probetas de los cuatro tipos de granitos, recogidas de un muro contiguo a la catedral que fue reconstruido durante las obras de intervención en el monumento. Este muro, orientado al sur, estaba construido con los mismos granitos de la catedral. De cada tipo de granito se utilizaron cuatro probetas cúbicas $(5 \times 5 \times 5 \mathrm{~cm})$ para los ensayos de absorción libre de agua; seis cubos (5x5x5 $\mathrm{cm}$ ) para los ensayos de densidad real y aparente; y cuatro prismas $(5 \times 5 \times 10 \mathrm{~cm})$ para el ensayo de capilaridad. Todas las probetas fueron previamente secadas en una estufa a una temperatura de $70{ }^{\circ} \mathrm{C}$ hasta alcanzar peso constante. La determinación de estas propiedades físicas se realizó siguiendo las normas UNE-EN 1925 (3), UNE-EN 1936 (4) y la norma del CNR-ICR Normal: 7/81 (5). La porosimetría se realizó mediante el porosímetro de mercurio en una sola muestra del granito de Lamares. Se seleccionó este granito debido a la marcada arenización que presenta en las columnas de la fachada principal (W) de la seo catedral de Vila Real. Debido a la fuerte desagregación granular, en estos pilares se recogieron cinco muestras de sales solubles en diferentes piedras graníticas de esta fachada mediante la técnica del "emplaste" y se realizó el análisis químico del extracto soluble mediante espectrometría de absorción molecular para $\left(\mathrm{SO}_{4}\right)^{2-},\left(\mathrm{Cl}^{-}\right)$, $\left(\mathrm{NO}_{3}{ }^{-}\right)$y $\left(\mathrm{NH}_{4}{ }^{+}\right)$, espectrometría de absorción atómica para $\mathrm{Ca}^{2+}$ y $\mathrm{Mg}^{2+}$ y espectrometría de emisión de llama para $\left(\mathrm{K}^{+}\right)$e $\left(\mathrm{Na}^{+}\right)$. Las sales solubles se recogieron después del proceso de limpieza.

\section{RESULTADOS Y DISCUSIÓN}

Todos los granitos tienen color amarillento y son moscovítico-biotíticos (Tabla I). En cuanto al tamaño de grano, el granito de Vale das Gatas (VG) es de grano medio y porfiroide, el granito de Paredes (Pa), grueso, el granito de Lamares (La), de medio a fino, y el granito de Benagouro $(\mathrm{Be})$, de grano medio (Tabla 1). Tienen como minerales esenciales cuarzo, feldespato potásico, plagioclasa (An < $5 \%$, moscovita y biotita. Como minerales accesorios se encuentra turmalina, apatito, circón y rutilo. Como minerales tardi a post-magmáticos aparecen fosfatos de la serie childrenita-eosforita (granito $\mathrm{Pa}$ ), determinados mediante análisis de microsonda electrónica, moscovita y clorita, éstos como resultado de la alteración heredada del granito. La caolinita, detectada mediante el microscopio electrónico de barrido, aparece como producto de alteración de 
Tabla 1 / Table 1

Principales características petrográficas de los granitos estudiados. Petrographic characteristics of studied granites.

\begin{tabular}{|c|c|c|c|c|}
\hline $\begin{array}{l}\text { Granito / } \\
\text { Granite }\end{array}$ & $\begin{array}{l}\text { Tamaño de grano y textura / } \\
\text { Size grain and texture }\end{array}$ & $\begin{array}{l}\text { \% Moscovita; \% Biotita / } \\
\% \text { Muscovite; \% Biotite }\end{array}$ & $\begin{array}{c}\text { Plagioclasa/FK / } \\
\text { Plagioclase/ K feldspar }\end{array}$ & $\begin{array}{c}\text { Alteraciones metasomáticas / } \\
\text { Metassomatic alteration }\end{array}$ \\
\hline Lamares (La) & $\begin{array}{l}\text { Medio a fino. } \\
\text { Hipidiomórfica granular / } \\
\text { Fine to medium. } \\
\text { Subhedral granular }\end{array}$ & $19 \pm 3 ; 3 \pm 1$ & $1.4 \pm 0.3$ & $\begin{array}{l}\text { Moscovitización de la plagioclasa } \\
\text { Albitización y caolinitización } \\
\text { del FK / Plagioclase } \\
\text { muscovitization Albitization and } \\
\text { kaolinization of K-feldspar }\end{array}$ \\
\hline Vale das Gatas (VG) & $\begin{array}{c}\text { Médio, porfiroide / Medium. } \\
\text { Porphyritic }\end{array}$ & $13 \pm 3 ; 6 \pm 2$ & $0.9 \pm 0.3$ & $\begin{array}{l}\text { Escasa albitización del FK y } \\
\text { moscovitización de la plagioclasa } \\
\text { Cloritización de la biotita / } \\
\text { Rare albitization of } K f \text { and } \\
\text { muscovitization of plagioclase } \\
\text { Cloritization of biotite }\end{array}$ \\
\hline Benagouro (Be) & $\begin{array}{l}\text { Medio. } \\
\text { Hipidiomórfica granular / } \\
\text { Medium. } \\
\text { Subhedral granular }\end{array}$ & $19 \pm 3 ; 3 \pm 1$ & $1.7 \pm 0.3$ & $\begin{array}{c}\text { Moscovitización de la plagioclasa } \\
\text { Caolinitización del FK / } \\
\text { Muscovitization of plagioclase } \\
\text { Kaolinization of } K f\end{array}$ \\
\hline Paredes $(\mathrm{Pa})$ & $\begin{array}{c}\text { Grueso. } \\
\text { Hipidiomórfica granular / } \\
\text { Coarse. } \\
\text { Subhedral granular }\end{array}$ & $16 \pm 4 ; 3 \pm 2$ & $1.4 \pm 0.7$ & $\begin{array}{c}\text { Caolinitización del FK } \\
\text { Microclinización y moscovitización } \\
\text { de la plagioclasa / Kaolinization } \\
\text { of Kf. Microclinization and } \\
\text { muscovitization of plagioclase }\end{array}$ \\
\hline
\end{tabular}

los feldespatos por reacciones de caolinización (hidrólisis), debilitando la microfábrica de los granitos y produciendo un acusado deterioro (6).

Las microfisuras juegan un papel esencial en el desarrollo de los procesos de degradación en las rocas graníticas. Por esto, el análisis de la red fisural es indispensable en los estudios de deterioro porque equivale a los caminos que potencialmente va a seguir el agua (7). El análisis de las fisuras en los cuatro granitos mediante el microscopio electrónico de barrido (MEB) (Figura 3), si bien fue semicuantitativo, permitió encontrar algunas diferencias en su red fisural, siendo el granito de grano medio a fino de Lamares (La), utilizado sobre todo en las columnas del pórtico, el que presenta más fisuras interconectadas, que permiten una transferencia rápida por capilaridad de las soluciones salinas provenientes del agua de lluvia y del agua subterránea, así como una rápida cinética de evaporación (8). El granito de Vale das Gatas (VG), que es el tipo de granito más abundante en la fachada, es el que presenta una menor densidad de microfisuración observándose, tal como sucede en los otros granitos, una mayor presencia de las fisuras intragranulares en los cristales de albita (Figura 3). Las fisuras intergranulares aparecen en todos los granitos, pero como se puede ver en la Figura 3, son más evidentes en el granito de Lamares, lo que, junto a su tamaño de grano más fino, facilitará su desagregación granular.

Los resultados de la determinación de las propiedades físicas de los granitos estudiados aparecen en la Tabla 2. Teniendo en cuenta que los granitos sanos suelen tener una porosidad abierta que no suele superar el $1 \%$, los granitos estudiados poseen una elevada porosidad (Tabla 2), como es frecuente en este tipo de rocas cuando se presentan más meteorizadas (8). El granito La, con una porosidad del 6,9\%, es el más alterado de los granitos estudiados, mientras que el granito VG es el menos poroso y el menos alterado, lo que está de acuerdo con las observaciones petrográficas y con las realizadas en el monumento. Algunos autores (9) han llegado a la conclusión de que las rocas graníticas más resistentes a los ensayos de alteración artificial acelerada son aquéllas con menor porosidad y con tamaño de grano más fino.

Tabla 2 / Table 2

Valor medio y desviación estándar de la densidad real, densidad aparente y porosidad abierta de los granitos estudiados. Mean and standard deviation of real density, apparent density, porosity and absorption of studied granites.

\begin{tabular}{|c|c|c|c|c|}
\hline Granitos / Granites & $\begin{array}{c}\text { Densidad real / } \\
\text { Real density } \\
\left(\mathbf{k g} / \mathbf{m}^{3}\right)\end{array}$ & $\begin{array}{c}\text { Densidad aparente / } \\
\text { Apparent density } \\
\mathbf{( k g / \mathbf { m } ^ { 3 } )}\end{array}$ & $\begin{array}{c}\text { Porosidad abierta / } \\
\text { Open porosity } \\
\mathbf{( \% )}\end{array}$ & $\begin{array}{c}\text { Absorción (a 72 horas) / } \\
\text { Absorption (72 hours) } \\
\text { (\%) }\end{array}$ \\
\hline Lamares (La) & $2660 \pm 4$ & $2480 \pm 11$ & $6.9 \pm 0.4$ & 2.15 \\
\hline Vale das Gatas (VG) & $2660 \pm 7$ & $2560 \pm 10$ & $4.0 \pm 0.4$ & 1.41 \\
\hline Benagouro (Be) & $2660 \pm 4$ & $2510 \pm 7$ & $5.7 \pm 0.2$ & 1.93 \\
\hline Paredes (Pa) & $2660 \pm 10$ & $2520 \pm 18$ & $5.4 \pm 0.7$ & 1.69 \\
\hline
\end{tabular}




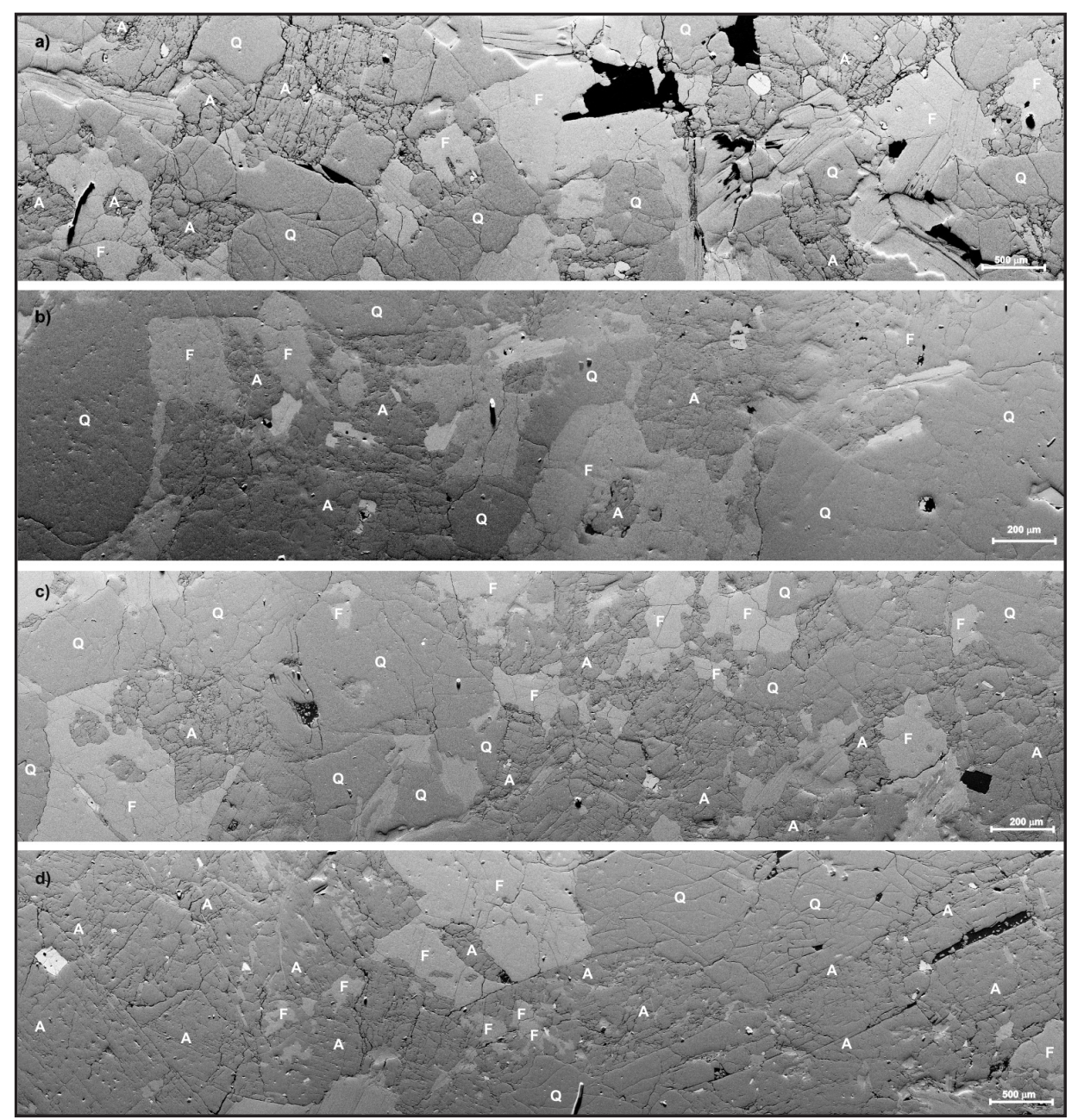

Figura 3. Imágenes de electrones retrodispersados (MEB) de la red de microfisuración a) granito de Vale das Gatas; b) granito de Paredes; c) granito de Benagouro; d) granito de Lamares.

Figure 3. Backscatered electron images on SEM showing intragranulares and intergranulares fissures a) granite of Vale das Gatas;

b) granite of Paredes; c) granito of Benagouro; d) granito of Lamares.

Con la evolución del proceso de meteorización, que corresponde a un aumento de la porosidad abierta, disminuye la densidad aparente. El granito VG, que presenta el mayor valor de densidad aparente, es el que posee la porosidad más baja (4\%).

La absorción de agua por inmersión suministra una buena información del grado de saturación máximo de la roca cuando esté bajo la acción de la lluvia, de la escorrentía superficial o de la ascensión capilar (10). Además, el estudio de la cinética de absorción nos da información respecto a la interconectividad y tortuosidad de los espacios vacíos, factor importante a la hora de absorber agua o de secar la roca puesta en obra. La absorción inicial, más rápida, está relacionada con la entrada de agua en los espacios vacíos mayores y bien interconectados, mientras que la absorción lenta, característica del final del ensayo, corresponde a la entrada de agua en los espacios vacíos más pequeños y de difícil acceso (11).
Los valores máximos alcanzados (en tres días) se incluyen en la Tabla 2. Observando el gráfico de la Figura 4 puede identificarse que la etapa de absorción rápida de agua, correspondiente a los 10 primeros minutos de inmersión, es similar para los cuatro granitos. La etapa de transición entre la absorción rápida y la lenta (curva casi horizontal), que casi no existe para el granito de Paredes $(\mathrm{Pa})$, es más larga en el granito VG. La absorción a las 72 horas es distinta para los cuatro granitos $y$, dado que la curva no es totalmente horizontal, o sea, no está estabilizada, puede decirse que el contenido en agua de saturación no se alcanzó y algo de aire quedó retenido en los espacios vacíos.

La absorción libre de agua determinada a las 24 horas presenta, como era de esperar, valores inferiores al contenido de agua de saturación (determinada al vacío) (Tabla 3). El grado de relleno de los poros $(\mathrm{Sr}$ ) refleja esta diferencia, pues expresa la relación entre la cantidad de agua libre absorbida (Ab) y el contenido máximo de absorción (Ws), expresado como porcentaje. 


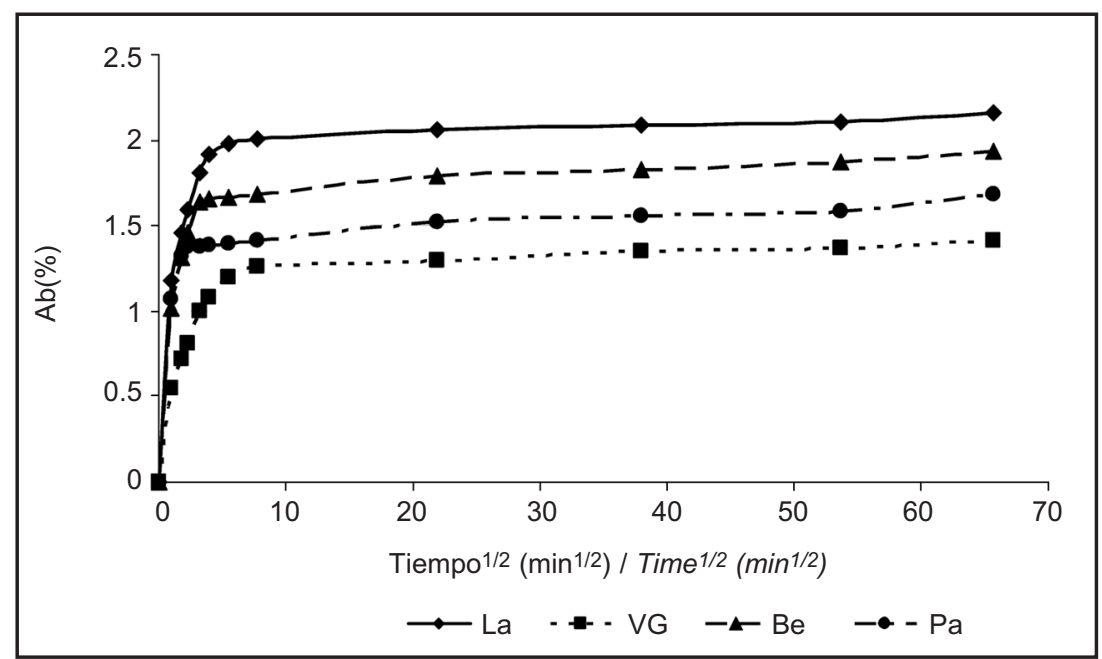

Figura 4. Evolución de la absorción de agua $\left(A_{b}\right)$ de los granitos estudiados durante el ensayo de absorción libre de agua. Figure 4. Evolution of the content of water absorption $\left(A_{b}\right)$ from the studied granites during the free water absorption along 72 hours.

Tabla 3 / Table 3

Valor medio y desviación estándar (\%) del contenido en agua de saturación, absorción libre a las 24 horas y grado de relleno de los poros a las 24 horas, de los granitos estudiados.

Mean and standard deviation (\%) of water content saturation, free absorption at 24 hours and level of pore filling at 24 hours, from granites.

\begin{tabular}{|c|c|c|c|}
\hline Granitos / Granites & $\begin{array}{c}\text { Contenido en agua de saturación / } \\
\text { Water content saturation }\end{array}$ & $\begin{array}{c}\text { Absorción libre a las 24 horas / } \\
\text { Free absorption at 24 hours }\end{array}$ & $\begin{array}{c}\text { Grado de relleno de los poros } \\
\text { a las 24 horas / } \\
\text { Level of pore filling at 24 hours }\end{array}$ \\
\hline Lamares (La) & $2.77 \pm 0.17$ & $2.09 \pm 0.02$ & 75.5 \\
\hline Vale das Gatas (VG) & $1.55 \pm 0.15$ & $1.35 \pm 0.04$ & 87.1 \\
\hline Benagouro (Be) & $2.26 \pm 0.08$ & $1.83 \pm 0.09$ & 81.0 \\
\hline Paredes (Pa) & $2.14 \pm 0.28$ & $1.56 \pm 0.03$ & 72.9 \\
\hline
\end{tabular}

No siempre la roca que presenta mayores valores de porosidad abierta es la que alcanza valores de absorción más altos, pues la tortuosidad de la configuración del sistema poroso va a determinar una mayor o menor dificultad de relleno de los espacios vacíos por el agua. Cuanto más lenta sea la absorción, menores serán los espacios vacíos y más tortuosa será su conexión. La falta de conectividad entre los poros obviamente reduce la porosidad abierta (12). El granito La, que presenta el mayor valor de porosidad abierta es también el que presenta el sistema poroso peor conectado y con predominio de espacios vacíos muy pequeños, de difícil acceso, donde el aire puede quedar fácilmente retenido, ya que es el granito con menor grado de relleno de los poros a las 72 horas (76\%). Además, el hecho de estar muy meteorizado puede haber conducido a la formación de minerales arcillosos, como la caolinita, que rellenan sus fisuras, limitando la circulación de agua, a pesar de que la fisuración de este granito es muy intensa.

Por otra parte, el granito VG, que es el menos poroso de los dos granitos estudiados, es el que posee el sistema poroso mejor conectado y con menor tortuosidad, ya que a las 24 horas del ensayo registró un valor de absorción de 1,35\%, correspondiente al $87 \%$ del contenido en agua de saturación. Al final del ensayo, a las 72 horas de inmersión, el grado de saturación medio ( $\mathrm{Sr}$ ) variaba desde un mínimo del $78 \%$, en el granito La, hasta un máximo del $91 \%$ en el granito de VG (Figura 5).

La porosimetría del granito La reveló que sólo el $18 \%$ de la porosidad abierta corresponde a macroporosidad, siendo la microporosidad del $82 \%$ (Tabla 4). La macroporosidad corresponde a poros entre 600 y 7,5 $\mu \mathrm{m}$ y la microporosidad, a poros entre 7,5 y 0,0035 $\mu \mathrm{m}$ (13).

En la Figura 6 puede observarse la curva acumulada del volumen de los diferentes rangos de tamaño de poros y su respectivo histograma. De ella se deduce que el tamaño de poro más abundante está alrededor de $1 \mu \mathrm{m}$.

Respecto a la capilaridad, hay que recordar que está condicionada, entre otros factores, por el tamaño de los espacios vacíos: la altura alcanzada será tanto mayor cuanto menor sea el diámetro de los canales de los poros $(13,14)$. Así, las rocas con los microporos más grandes son las que presentan generalmente la mayor transferencia capilar (13). Este parámetro depende además de los minerales constituyentes, de la pureza del agua o de la humedad relativa (14). 


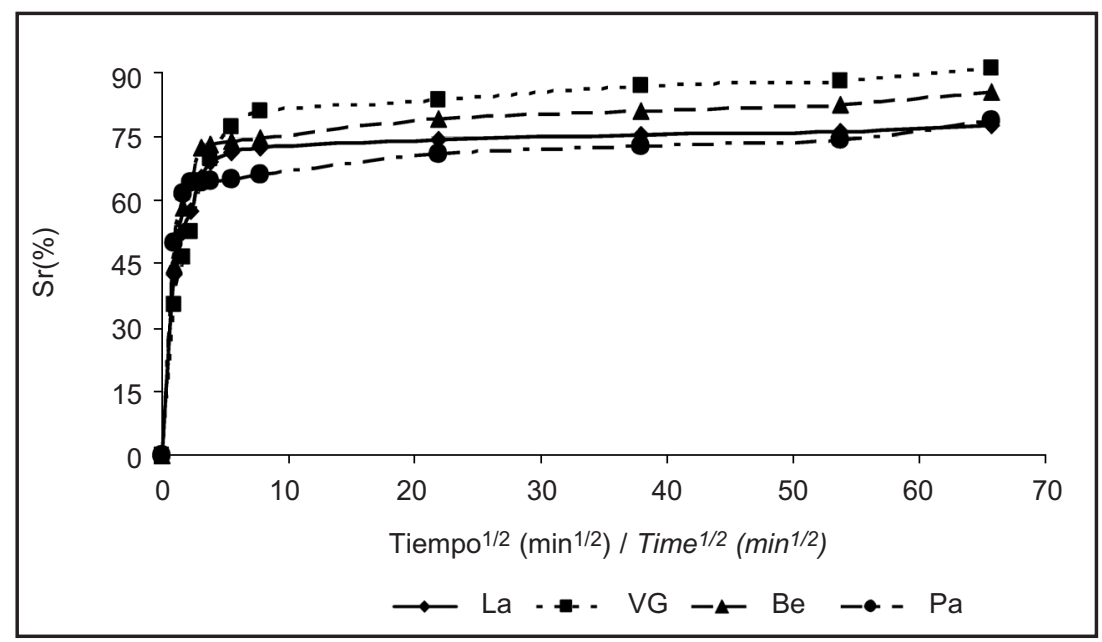

Figura 5. Variación del grado de saturación (Sr) de los granitos estudiados durante el ensayo de absorción libre de agua. Figure 5. Evolution of the saturation degree ( $\mathrm{Sr}$ ) from the studied granites during the free water absorption.

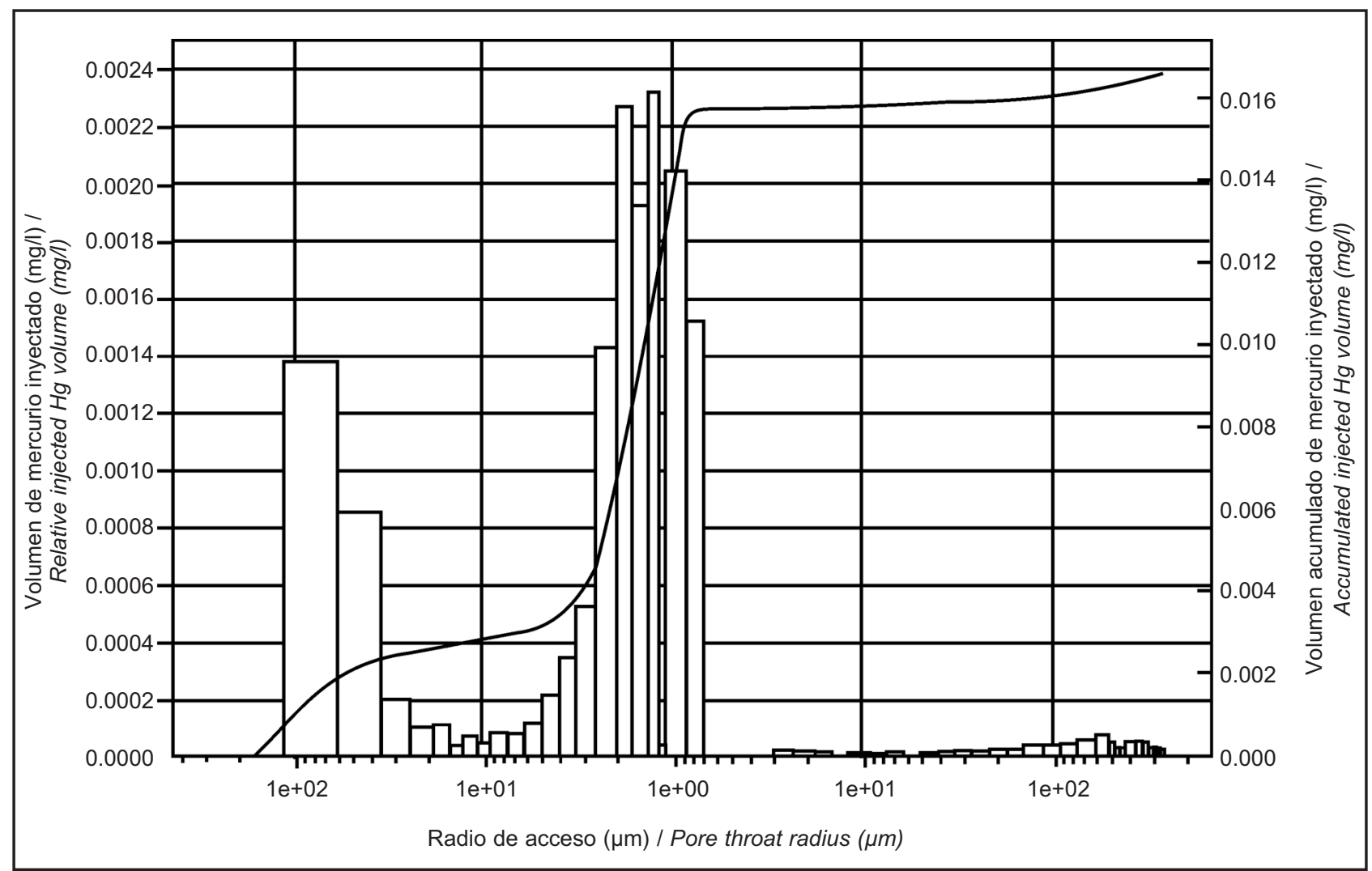

Figura 6. Curva acumulada del volumen de los diferentes rangos de tamaño de poro y su respectivo histograma para el granito de Lamares.

Figure 6. Volume accumulated curve of the various pore sizes and its respective histogram for Lamares granite.

Tabla 4 / Table 4

Resultados de la porosimetría de mercurio del granito de Lamares.

Mercury porosimetry of the granite of Lamares.

\begin{tabular}{|c|c|c|c|c|c|}
\hline $\begin{array}{c}\text { Granito / } \\
\text { Granite }\end{array}$ & $\begin{array}{c}\text { Porosidad de mercurio / } \\
\text { Mercury porosimetry }\end{array}$ & $\begin{array}{c}\text { Macroporosidad / } \\
\text { Macroporosity }\end{array}$ & $\begin{array}{c}\text { Microporosidad / } \\
\text { Microporosity }\end{array}$ & $\begin{array}{c}\text { Radio medio de acesso } \\
\text { de poros / Medium pore } \\
\text { acess radius }(\boldsymbol{\mu m})\end{array}$ & $\begin{array}{c}\text { Superficie específica / } \\
\text { Specific surface } \\
\left(\mathbf{m}^{2} / \mathbf{g}\right)\end{array}$ \\
\hline Lamares (La) & 4.08 & $0.73(18 \%)$ & $3.35(82 \%)$ & 0.12 & 0.28 \\
\hline
\end{tabular}


Los fenómenos de capilaridad se manifiestan sobre todo en las zonas inferiores de los edificios, dado que la humedad del suelo se transfiere a los materiales más próximos, originando variaciones de la cantidad de agua y de sales disueltas, que favorecen la degradación progresiva de las rocas (13). La cristalización de sales solubles en rocas porosas ha sido ampliamente aceptada como uno de los mecanismos más importantes de deterioro del patrimonio arquitectónico construido con materiales pétreos (12) y la temperatura, a su vez, es uno de los factores que controla este proceso $(15,16)$. Sin embargo, hay otros factores que influyen en los daños causados por la cristalización de sales, especialmente la cantidad y tamaño de los poros, la naturaleza de la sal, la facilidad de saturación de la solución y la resistencia del material a la cristalización (12).

En la Figura 7 se observa que el comportamiento de los granitos La, Be y Pa son muy similares durante la primera hora de ensayo, mientras que el granito VG tiene una cinética de absorción de agua por capilaridad más lenta. A partir de la primera hora (Figura 7) los tres granitos absorben agua con velocidades distintas. Así, el granito La, que presenta, como se ha mencionado anteriormente, el mayor valor de porosidad abierta y una alta microporosidad, fue el que absorbió más agua por transferencia capilar mientras que el granito VG, con una porosidad abierta de 4,0\%, fue el que menos absorbió.

\section{PRINCIPALES PATOLOGÍAS DE LAS ROCAS GRANÍTICAS}

La ciudad de Vila Real está localizada en una zona poco industrial, por lo que la contaminación atmosférica puede considerarse baja. Las principales fuentes de contaminantes son la calefacción doméstica y la combustión de carburantes de los vehículos de transporte, por lo que su incidencia sobre las piedras de la catedral no es muy importante. Las principales patologías que se aprecian en la catedral están más relacionadas con los factores climáticos y los fenómenos de ascensión capilar de agua del subsuelo que con los contaminantes atmosféricos.

Así se han encontrado distintas formas de alteración, como las desplacaciones y escamas (relacionadas con fenómenos de hielo-deshielo o de cristalización de sales), pátinas negras (debidas al depósito de cenizas, humos, polvo, etc., sobre la superficie de las piedras), costras negras (muy escasas y normalmente con presencia de yeso) así como otras formas de alteración física (fisuras de origen mecánico, fruto de la concentración de tensiones en algunas zonas) y biológica (crecimiento de algas, líquenes y plantas superiores sobre o entre los sillares de granito). De estas formas de alteración, sólo las costras y las pátinas negras pueden estar relacionadas con la contaminación ambiental, aunque, por su posición en la parte inferior del edificio, han sido favorecidas por el fenómeno de capilaridad. En la Figura 8 ( $a$ y b) se puede ver la distribución de las formas de alteración a los lados de la puerta principal de la catedral, que, a su vez, puede relacionarse con la distribución de los diferentes tipos de granitos de la Figura 2.

Pero en la fachada principal de la catedral, la forma de alteración más destacable es la desagregación granular, relacionada con la presencia de sales solubles en el agua que penetra en las piedras. De sobra es conocido que el crecimiento de cristales de sal en los espacios vacíos de las rocas es una de las principales causas de deterioro en los monumentos hechos con piedra (16-21).

Teniendo en cuenta que el lateral este de la catedral está recubierto de mortero de cemento y que de los otros tres laterales, es la fachada oeste (donde se encuentra la puerta principal) la que presenta un mayor grado de arenización, se ha realizado la extracción de cinco muestras

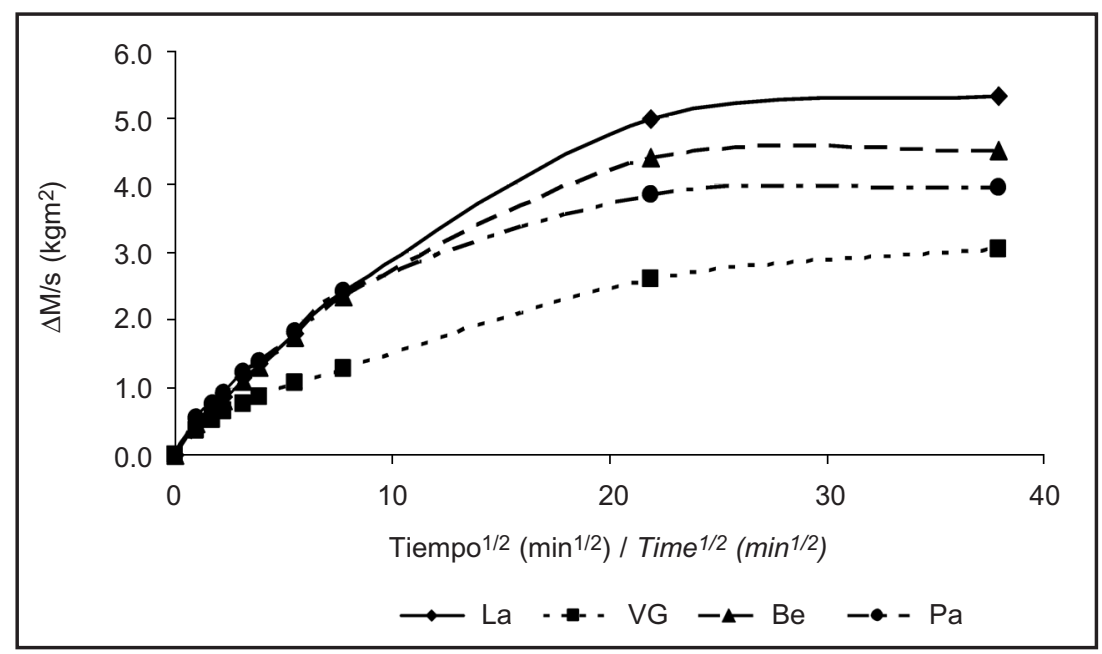

Figura 7. Variación del peso de agua por unidad de superficie durante el ensayo de capilaridad. Figure 7. Evolution of the weight samples per surface unit during the cappilarity test along 24 hours. 


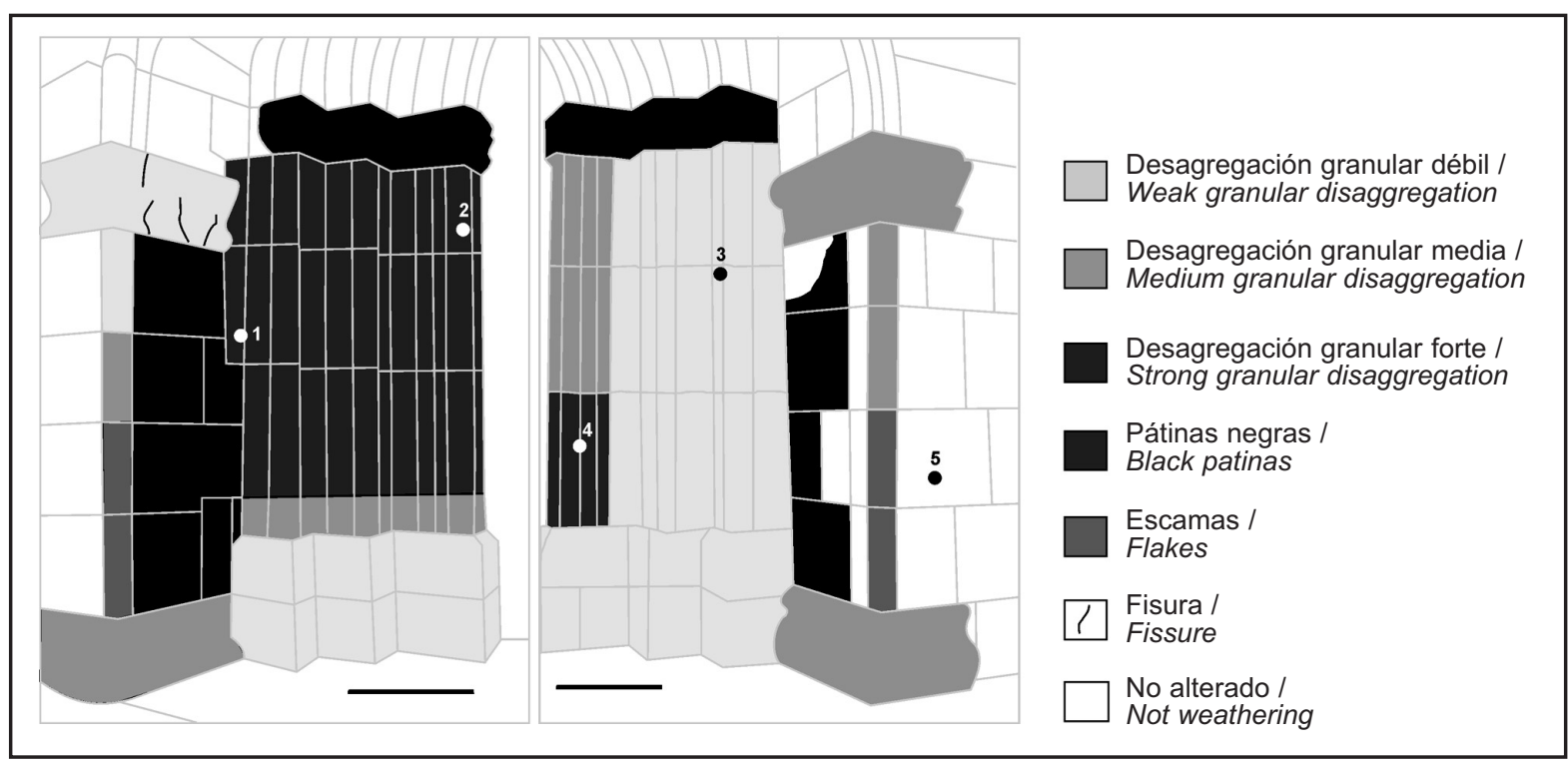

Figura 8. Cartografía de las formas de alteración a ambos lados de la puerta principal de la catedral: a) lado izquierdo; b) lado derecho. 1, 2, 3, 4, 5 - Localización de los puntos donde se recogieron sales solubles.

Figure 8. Deterioration mapping on the main front of the cathedral: a) left side; b) right side. 1, 2, 3, 4, 5 - Localization of points where soluble salts were collected.

de sales por el método del emplaste en esta fachada. La localización de los puntos de extracción aparece en la Figura 8. Como se puede apreciar, la mayor parte de las extracciones se realizaron en el granito de Lamares, que es el más arenizado, especialmente en las columnas situadas a la izquierda del pórtico.

Las sales solubles que aparecen en las columnas (muestras 1, 2, 3, 4) y en los muros (muestra 5) de la catedral son principalmente cloruro de sodio y nitrato de calcio (Tabla V). La columna donde se recogió la muestra 2 es la más arenizada y presenta una gran cantidad de sales solubles. Las sales solubles revelan así la presencia de un sistema salino dominado por cloruros de sodio (muestras 2 y 4 ) y, además, un sistema salino asociado con la polución orgánica humana, evidenciado por la abundancia de amonio y nitrato (muestra 4, que se encuentra más próxima al suelo). Aunque algunas sales, como la halita, silvina, sulfatos de calcio y potasio y otras, pueden proceder de los excrementos de las aves (pueden contener un $4 \%$ de sales solubles) (17) y la presencia de cloruros haya sido también atribuida a la acción marina (sprays de agua marina) incluso a decenas de kilómetros del mar (22), en el caso de la catedral de Vila Real parece que procedan de las aguas subterráneas que suben por capilaridad por sus paredes. En la Figura 1 se puede apreciar que la parte inferior de las paredes de la catedral, de un tono más oscuro, muestra evidencias de la existencia de este fenómeno. Por otra parte, algunos autores (23) han mencionado que las aguas procedentes de suelos con asentamientos humanos suelen estar enriquecidos en nitratos (procedentes de microorganismos derivados de desechos orgánicos) y cloruros (por el consumo de cloruro sódico). Además, los nitratos y sulfatos suelen ser abundantes en terrenos dedicados a la agricultura.

Analizando al MEB algunas muestras de producto de alteración del granito recogidas donde la muestra 2, puede confirmarse la presencia de halita en forma de

Tabla 5 / Table 5

Contenido en sales solubles $(\mathrm{mg} / \mathrm{l})$ en el estracto soluble de las muestras estudiadas. Content of soluble salts $(\mathrm{mg} / \mathrm{l})$ on the soluble extract of granites.

\begin{tabular}{|c|c|c|c|c|c|c|c|c|c|}
\hline $\begin{array}{c}\text { Muestra/ } \\
\text { Sample }\end{array}$ & $\begin{array}{c}\text { Conductividad / } \\
\text { Condutivity } \\
\text { Ds.m-1 }\end{array}$ & $\mathbf{N H}_{4}{ }^{+}$ & $\mathbf{M g}^{2+}$ & $\mathbf{C a}^{2+}$ & $\mathbf{K}^{+}$ & $\mathbf{N a}^{+}$ & $\mathbf{C l}^{-}$ & $\mathbf{S O}_{\mathbf{4}^{2-}}$ & $\mathbf{N O}^{-}$ \\
\hline $1(\mathrm{G} \mathrm{Pa})$ & 0.178 & 0.05 & 1.5 & 25.5 & 6 & 12 & 10.95 & 3.60 & 5.71 \\
\hline $2(\mathrm{G} \mathrm{La})$ & 3.9 & 0.08 & 13.75 & 50 & 32 & 134 & 166.46 & 18.16 & 111.76 \\
\hline $3(\mathrm{G} \mathrm{La})$ & 0.297 & 0.02 & 4 & 40.8 & 12 & 16 & 24.47 & 8.29 & 18.96 \\
\hline $4(\mathrm{G} \mathrm{La})$ & 7.96 & 1.24 & 16.25 & 65 & 125 & 125 & 170.63 & 20.23 & 367.33 \\
\hline $5(\mathrm{G} \mathrm{Pa})$ & 0.161 & 0.09 & 5.25 & 40.8 & 7 & 7 & 13.81 & 3.59 & 14.64 \\
\hline
\end{tabular}




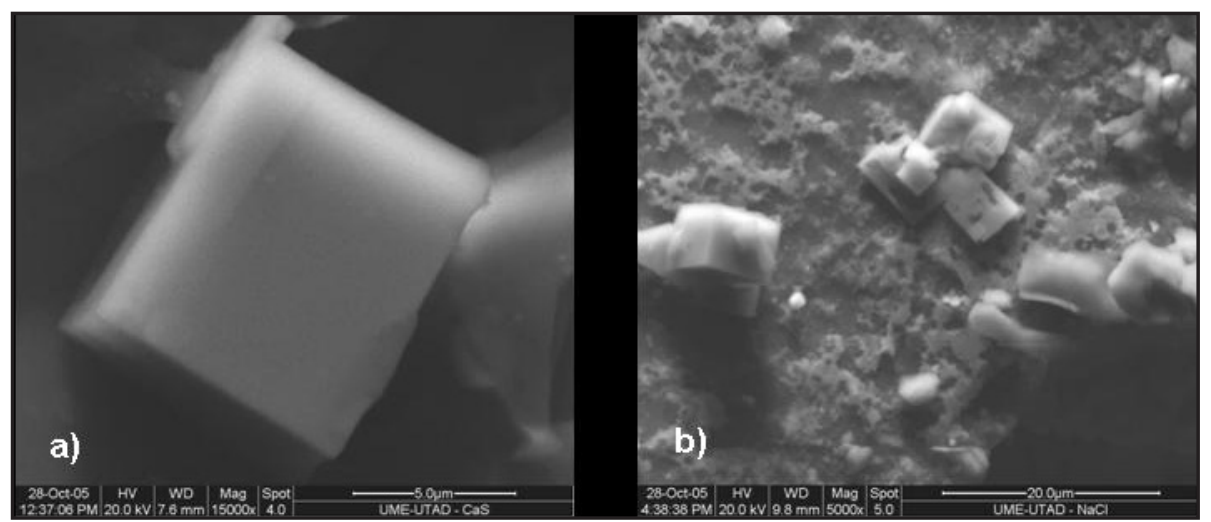

Figura 9. Desagregación granular: a) y b) halita en forma de cristales cúbicos perfectos. Figure 9. Granular desintegrationr: a) and b) perfect cubic crystals of halite.

cristales cúbicos perfectos (Figura 9). El yeso, bajo la forma de cristales pseudoexagonales a veces con bordes redondeados, apenas aparece y sólo se identificó en una muestra de esta columna recogida en la parte más inferior. También se han encontrado cristales de calcita y óxidos de hierro.

Es fácil de entender que la presencia de sales en el granito de Lamares ha originado un aumento de su deterioro. Como se ha mencionado previamente, la desagregación granular más intensa se localiza en los sillares del granito de Lamares situados a la izquierda de la puerta principal (fachada oeste). Por una parte, existe un condicionamiento litológico: el granito de Lamares es la roca con mayor porosidad abierta y mayor absorción de agua; además, la porometría de mercurio ha puesto de manifiesto el pequeño tamaño de sus espacios vacíos, lo que lo hace más vulnerable a la heladicidad y al deterioro por cristalización de sales. Por otra parte, este granito es el de tamaño de grano más fino y el que tiene una mayor fisuración intergranular, lo que contribuye a una mayor disgregación granular potencial. En rocas graníticas ornamentales parecidas a las de la catedral de Vila Real (con una porosidad abierta inferior al 5\%) algunos autores (21) han llegado a la conclusión de los granitos de grano grueso y de baja porosidad son los que ven su red fractográfica más afectada por los ciclos de cristalización de sales y que los más ricos en micas y plagioclasas pierden con más facilidad el pulimento.

Además de esta influencia litológica existe otro factor importante que afecta a la desagregación granular, que es la localización de los materiales en el edificio. La fachada principal de la catedral está orientada al oeste, por lo que, a partir de la tarde, el sol insolará los sillares de esta fachada, lo que hará que el agua de capilaridad (con las sales solubles) tienda a evaporarse, precipitando las sales solubles en los espacios vacíos más superficiales del granito, contribuyendo a su desagregación granular. No obstante, si nos fijamos en la Figura 8, las columnas del granito de Lamares que están a la izquierda de la entrada están más arenizadas que las de la derecha, debido a que la insolación es mayor en la parte izquierda que en la derecha; el pórtico no es plano y los pilares de la derecha están orientados al noroeste, mientras que los de la izquierda, lo están al suroeste, más soleado. Por eso, las mayores temperaturas alcanzadas en las columnas de la izquierda debido a su mayor tiempo de exposición al sol, producen una mayor evaporación del agua de capilaridad, a la vez que han podido influir en la precipitación, presión de cristalización y expansión térmica de las sales $(16,24)$. Recientemente, algunos autores (25) han señalado que las fluctuaciones de humedad relativa parecen ser en gran parte responsables del deterioro producido por sales de sulfato de magnesio en probetas de calizas en ensayos de laboratorio.

\section{CONCLUSIONES}

La piedra utilizada en la construcción del monumento de la seo catedral de Vila Real es de origen local y corresponde al menos a cuatro tipos de granitos distintos, que afloran en las inmediaciones de la ciudad. El granito con más problemas de arenización es el granito de Lamares, que es el granito de grano más fino, más alterado petrográficamente, con mayor porosidad (en su mayor parte microporosidad) y con una microfisuración intergranular muy evidente; además es el que absorbió más agua por transferencia capilar y el que presenta mayor cantidad de sales solubles.

La desagregación granular es la patología dominante, provocando daños significativos en la zona alrededor de la entrada principal de la catedral, siendo la responsable de la pérdida de definición de las columnas situadas a ambos lados. Este tipo de deterioro está relacionado con el ascenso y cristalización de soluciones salinas 
procedentes del subsuelo junto con los efectos de la insolación.

La parte de la izquierda de la entrada principal posee un grado de deterioro mayor que la de la derecha. En nuestra opinión, este hecho se debe a la diferente insolación que reciben ambas partes, mayor en la izquierda, influyendo sobre la evaporación de las soluciones salinas y, por tanto, en la cantidad de sales cristalizadas. Esto apoya la importancia de la distribución de la temperatura en las rocas como un factor que influye en la velocidad de deterioro de las rocas, ya puesto de manifiesto por otros autores $(15,16)$.
Las sales solubles que aparecen en las paredes del monumento son mayoritariamente cloruros y nitratos de calcio y sodio. También se han encontrado contenidos menores de sulfato de calcio y sales de amonio. Dichas sales parecen proceder de las aguas subterráneas que ascienden por capilaridad por las paredes del edificio. Mientras no se solucione el problema de ascensión capilar, mediante la impermeabilización de los cimientos (aunque sea una solución un tanto costosa), cualquier método de restauración que se tome no acabará con el problema y el edificio continuará su proceso de deterioro.

\section{BIBLIOGRAFÍA}

(1) Matos, V.: "A geologia da região de Vila Real". Tese de Doutoramento, Universidade de Trás-os-Montes e Alto Douro, Vila Real (1991), p. 312.

(2) Peixoto, J. C. C.; Pinho, M. M.; Vinagre, P. J. S.: "Monitorização da qualidade do ar em Vila Real". Relatorio no publicado, Universidade de Trás-os-Montes e Alto Douro, Vila Real (2004), p. 42.

(3) Norma UNE-EN 1936: "Determinación de la densidad real y aparente y de la porosidad aberta y total". AENOR, Madrid (1999).

(4) Norma UNE-EN 1925: "Absorción de agua por Capilaridad". AENOR, Madrid (1999).

(5) Norma ICR 7/81: "Absorção de água por imersão total - capacidade de embebição". ICR - Instituto Central de Restauro (1981).

(6) Schiavon, N.: "Kaolinisation of granite in an urban environment". Env. Geol., vol. 52 (2007), pp. 399-407. doi:10.1007/s00254-006-0473-0

(7) Ordaz, J.; Pérez-Ortiz A.; Esbert R. M.; Martínez-Nistal, A.; Alonso, F. J.: "Study of the granite crack network by means of digital image processing". Proceedings of the EC Workshop on Degradation and conservation of granitic rocks in monuments. Protection and Conservation of European Cultural Heritage, Research Report n05, 1996, pp. 249-254.

(8) Begonha, A.: "Meteorização do granito e deterioração da pedra em monumentos e edifícios da cidade do Porto". Tese de Doutoramento, Universidade do Minho, Braga (2001), p. 393.

(9) Rivas Brea, T.; Prieto Lamas, B.; Silva Hermo, B.: "Ensayos de alteración artificial aplicados a rocas graníticas". Mater. Construcc., vol. 58, n. 289-290 (2008), pp. 179-189. doi:10.3989/mc.2008.v58.i289-290.80

(10) Aires-Barros, L.: "As rochas dos monumentos portugueses, tipologias e patologias". Instituto Português do Património Arquitectónico. Ministério da Cultura, Lisboa, volume I (2001), p. 335.

(11) Sousa, L. M. O.: "Estudo da fracturação e das características físico-mecânicas de granitos da região de Trás-os-Montes com vista à sua utilização como rocha ornamental". Tese de Doutoramento. Universidade de Trás-os-Montes e Alto Douro. Vila Real (2000<), p. 358.

(12) Benavente, D.; Cueto, N.; Martínez-Martínez, J.; García del Cura, M. A; Cañaveras, J. C.: "The influence of petrophysical properties on the salt weathering of porous building rocks". Env. Geol., vol. 52 (2007), pp. 215-224. doi:10.1007/s00254-006-0475-y

(13) Esbert, R. M.; Ordaz, J., Alonso, F. J.; Montoto, M.; González Limón, T.; Ballester, M. A. B.: "Manual de diagnosis y tratamiento de materiales pétreos y cerámicos". Collegi.d 'Aparelladors i Arquitectes Tècnics de Barcelona, Barcelona (1997), p. 139.

(14) Montoto, M.: "Petrophysics at the rock matrix scale: hydraulic properties and petrographic interpretation". Publicaciones técnicas Enresa (2003), p. 291.

(15) Steiger, M.: "Crystal growth in porous materials - II: influence of crystal size on the crystallization pressure". J Cryst Growth, vol. 282 (2005), pp. 470-481. doi:10.1016/j.jcrysgro.2005.05.008

(16) Gómez-Heras, M.; Fort, R.: "Patterns of halite ( $\mathrm{NaCl})$ crystallisation in building stone conditioned by laboratory heating regimes". Env. Geol., vol. 52 (2007), pp. 259-267. doi:10.1007/s00254-006-0538-0

(17) Gómez-Heras, M.; Benavente, D.; Buergo, M. A., Fort, R.: "Soluble salts minerals from pigeon droppings as potential contributors to the decay of stone based Cultural Heritage". Eur. J. Min. 16 (2004), pp. 505-509. doi:10.1127/0935-1221/2004/0016-0505

(18) Angeli, M.; Bigas, J.; Benavente, D.; Menéndez, B.; Hébert, R.; David, C.: "Salt crystallization in pores: quantification and estimation of damage". Env. Geol., vol. 52 (2007), pp. 205-213. doi:10.1007/s00254-006-0474-z

(19) Silva, B.; Rivas, T.; Prieto, B.; Auger, F.: "Soluble Salts in Granitic Monuments: Origin and Decay Effects". In: Applied Study of Cultural Heritage and Clays (2003), pp. 113-131.

(20) Charole, A. E.; Pühringer, J.; Steiger, M.: "Gypsum: a review of its role in the deterioration of building materials". Env. Geol., vol. 52 (2007), pp. 339-352. doi:10.1007/s00254-006-0566-9

(21) Alonso, F. J.; Vázquez, P.; Esbert, R. M.; Ordaz, J.: "Durabilidad de granitos ornamentales: valoración de los daños inducidos por el ensayo de cristalización de sales". Mater. Construcc., vol. 58, n. 289-290 (2008), pp. 191-201. doi:10.3989/mc.2008.v58.i289-290.78 
(22) Silva, B.; Rivas, T.; García-Rodeja Prieto, B.: "Distribution of ions of marine origin in Galicia (NW Spain) as a function of distance from the sea". Atmospheric Environment, 41 (2007), pp. 4396-4407. doi:10.1016/j.atmosenv.2007.01.045

(23) Grossi, C.: "Cristalización de sales en rocas monumentales porosas y su auscultación mediante emisión acústica". Tesis Doctoral. Departamento de Geología, Universidad de Oviedo (España) (1992), p. 261.

(24) Smith, B. J.; Warke, P. A.; McGreevy, J. P.; Kane, H. L.: "Salt-weathering simulations under hot desert conditions: agents of enlightenment or perpetuators of preconceptions?". Geomorph., 67 (2005), pp. 211-227. doi:10.1016/j.geomorph.2004.03.015

(25) López-Arce, P.; Doehne, E.; Martin, W., Pinchin, S.: "Sales de sulfato magnésico y materiales de edificios históricos: simulación experimental de laminaciones en calizas mediante ciclos de humedad relativa y cristalización de sales". Mater. Construcc., vol. 58, n. $289-290$ (2008), pp. 125-142. 